\title{
Síntese e Caracterização Físico-Química de Derivados de Óleos Vegetais Nativos para Uso como Óleo Base Lubrificante
}

\author{
Amanda S. Almeida, Paulo R. R. de Matos \& José Roberto S. Politi
}

\begin{abstract}
Atualmente existe uma grande preocupação na busca de fontes alternativas de produção que degradem o meio ambiente o mínimo possível. Nesse sentido, há uma disposição muito grande em reduzir a dependência da sociedade atual dos derivados de petróleo, substituindo estes por materiais renováveis e sustentáveis. A partir dessa visão ambiental e sustentável, este trabalho avalia o uso de óleo vegetal e alguns de seus derivados como alternativa aos óleos lubrificantes de matriz mineral. O óleo vegetal estudado nesse trabalho foi o óleo de Canola que é produzido em abundância no Brasil. As propriedades físico-químicas investigadas para avaliar a viabilidade deste óleo e seus derivados como base lubrificante foram índice de acidez, viscosidade cinemática e estabilidade oxidativa. O diol e o diéster produzidos a partir do óleo de Canola melhoraram a viscosidade e a estabilidade oxidativa em comparação com o óleo natural, mas mostrou pequeno aumento na acidez. Essas características indicam que o óleo de Canola "in natura" e os derivados que foram produzidos a partir dele podem se tornar viáveis alternativa aos óleos lubrificantes de matriz mineral.
\end{abstract}

\section{Palavras-chave: Biolubrificantes; Óleo Vegetal; Estabilidade Oxidativa.}

Nowadays there is great concern in the search for alternative sources of production that degrade the environment as little as possible. In this sense, there is a very large provision to reduce the dependence of current society on oil products, replacing these derivatives with renewable and sustainable materials. From this environmental and sustainable view, this work evaluates the use of vegetable oil and some of its derivatives as an alternative to lubricating mineral matrix oils. The oil studied was Canola oil produced abundantly in Brazil. The physicochemical properties investigated to evaluate the viability of this oil and its derivatives as a lubricants were acidity index, kinematic viscosity and oxidative stability. Diol and diester produced from Canola oil improved viscosity and oxidative stability compared to in nature oil but showed a small increase in acidity. These characteristics indicate that the Canola oil and its derivatives can become a viable alternative to mineral matrix lubricanting.

Keywords: Biolubricants; Vegetable Oil; Oxidative Stability. 


\section{Introdução}

Os óleos lubrificantes são compostos, fabricados com intuito de reduzir o ruído, calor e desgaste entre componentes móveis de equipamentos, aumentando sua vida útil. Cotidianamente pode ser observada a utilização desses óleos em diversas áreas, tanto industrial como automotiva, tendo como principal fonte o petróleo.

A fim de reduzir os impactos causados por essa fonte não renovável e pelo crescente esgotamento das reservas de petróleo, pesquisas têm sido desenvolvidas com intuito de buscar fontes alternativas (sustentáveis) de produção de óleo, que possuam a mesma eficácia dos óleos atualmente produzidos e comercializados $[4,8]$. Uma delas se baseia no uso de lubrificantes à base de óleo vegetal em lugar dos lubrificantes à base de petróleo, já que muitos biolubrificantes são biodegradáveis e não tóxicos, apresentando baixo risco de contaminação para o meio ambiente ou operadores.

Para que o óleo seja considerado um lubrificante, este deve apresentar características físico-químicas específicas, como: viscosidade cinemática, índice de viscosidade, ponto de fulgor, índice de acidez, ponto de fluidez, estabilidade oxidativa, volatilidade e etc[4]. Segundo Borugadda, Somidi eDalai (2017)[10] asreações utilizadas para remover as insaturações presentes nas moléculas e que reduzem significativamente sua estabilidade, inserem funcionalidades que melhoram as propriedades lubrificantes tais como a fluidez a baixas temperaturas, viscosidade, além da própria estabilidade.

O objetivo desse trabalho é verificar o potencial do óleo de canola e de seus derivados para atuarem como lubrificantes, pela análise do índice de acidez e a viscosidade. Os derivados foram produzidos por meio das reações descritas por Borugadda,Somidi e Dalai (2017)[10].

Inicialmente foram realizadas reações para obtenção do epóxido a partir do óleo de Canola com 0,25 mols (64,6 gramas) de duplas ligações do óleo de Canola, 0,25 mols $(11,5 \mathrm{ml})$ de ácido fórmico, $1 \mathrm{~mol}(102 \mathrm{ml})$ de peróxido gotejado lentamente, por cerca de 30 minutos. Após isso a temperatura do meio reacional foi aumentada para $60^{\circ} \mathrm{C}$, durante 8 horas.

\section{Metodologia}

Para obtenção do diol, a reação continuou com os mesmos reagentes sob temperatura de $80{ }^{\circ} \mathrm{C}$ por 14 horas. Foi feita a purificação com a água e acetato de etila, secagem com sulfato de sódio e a rotovaporização,

A obtenção do diéster se deu a partir do diol. A reação foi realizada em temperatura ambiente, durante 30 minutos. Acrescentou-se 0,1 mol (33,6 gramas) do diol, $100 \mathrm{ml} \mathrm{de}$ diclorometano, 1,6 g de DMAP, $16 \mathrm{ml}$ de trietilamina e 18 $\mathrm{ml}$ de anidrido acético. Após o fim da reação a mistura foi então rotovaporizada. Um fluxograma da metodologia é apresentado na figura 1 .

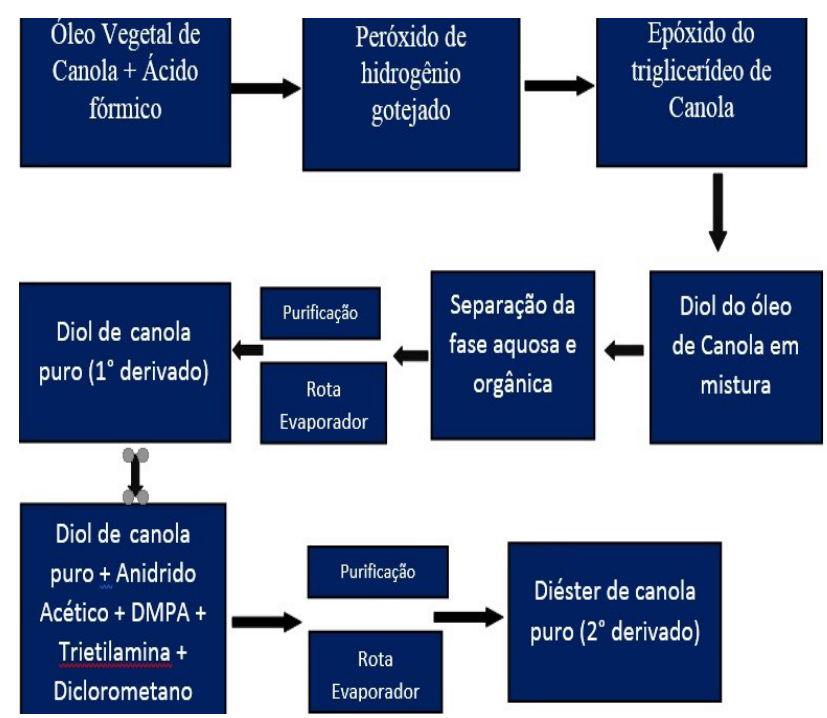

Figura 1. Metodologia utilizada no processo de produção dos derivados do óleo de canola

\section{Resultados e Discussão}

\section{CARACTERIZAÇÃO DO ÓLEO DE CANOLA E SÍNTESE DOS DERIVADOS}

Inicialmente foram medidas as propriedades físico- 
químicas para o óleo de Canola in natura. Os resultados obtidos para a viscosidade cinemática a $40{ }^{\circ} \mathrm{C}$ (Visc $40 \mathrm{oC}$ ) e $100{ }^{\circ} \mathrm{C}$ (Visc $100 \mathrm{oC}$ ), índice de viscosidade (IV), ponto de fulgor, ponto de fluidez, volatilidade (Vol) e estabilidade oxidativa (EOxi) são apresentados nas tabelas 1 e 2.

Tabela 1. Parâmetros físico-químicos do óleo de Canola in natura (dados da Agência Nacional de Petróleo, Gás Natural e Biocombustíveis (ANP))

\begin{tabular}{|c|c|c|c|c|c|c|}
\hline $\begin{array}{c}\text { Visc } \\
40 \mathrm{oC} \\
(\mathrm{mm} 2 / \mathrm{s})\end{array}$ & $\begin{array}{c}\text { Visc } \\
100 \mathrm{oC} \\
(\mathrm{mm} 2 / \mathrm{s})\end{array}$ & IV & $\begin{array}{c}\text { Fulgor } \\
\left({ }^{\circ} \mathrm{C}\right)\end{array}$ & $\begin{array}{c}\text { Fluidez } \\
\left({ }^{\circ} \mathrm{C}\right)\end{array}$ & $\begin{array}{c}\text { Vol } \\
(\%)\end{array}$ & $\begin{array}{c}\text { EOxi } \\
(\mathrm{min})\end{array}$ \\
\hline 36,51 & 8,25 & 211 & 300,1 & -15 & 0,6 & 14,75 \\
\hline
\end{tabular}

Foi feito o espectro RMN H1 do óleo de Canola in natura (figura 1) para caracterizar o óleo e determinar seu índice de insaturação, massa molar e a massa presente em 1 mol de insaturações, informações relevantes para a próxima etapa de síntese dos derivados

O espectro de RMN H1, apresentado a seguir, foi obtido no equipamento de $300 \mathrm{MHz}$ e tratado com o programa Mestre $\mathrm{C}$, com os valores referenciados pela integral dos picos:

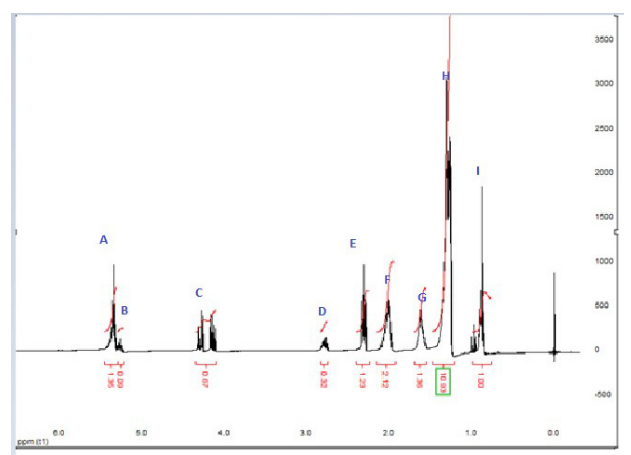

Figura 2. Espectro RMN-H óleo de Canola.
Cada pico foi relacionado com um ambiente químico apresentado na figura 3:

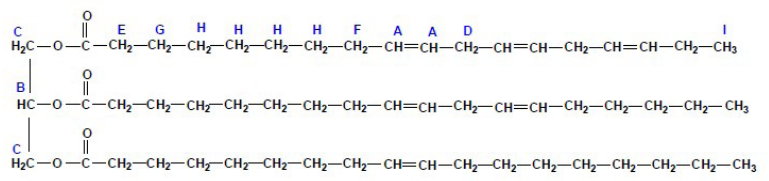

Figura 3. Atribuição dos picos de RMN H1 de um óleo vegetal na estrutura química de um triglicerídeo

Baseados nos picos do espectro, e na técnica utilizada por Miyake et al (1998)[12], pôde-se calcular a Massa molar e o indice de instauração do óleo de canola:

- Índice de insaturação: 3,71

- Massa molar: 958,07g/mol

- Massa presente em $1 \mathrm{~mol}$ de insaturações: 258,31g

Nessa etapa do trabalho, foi realizada a síntese do diol e do diéster do óleo de Canola. A síntese desses compostos parte do epóxido, que foi inicialmente produzido seguindo os procedimentos descritos na metodologia experimental.

O diol foi obtido pela reação de hidratação do epóxido. O anel epóxido é aberto com molécula de água, de modo que onde se tinha uma insaturação agora tem-se dois grupos $\mathrm{OH}$ em cada carbono que fazia a dupla ligação.

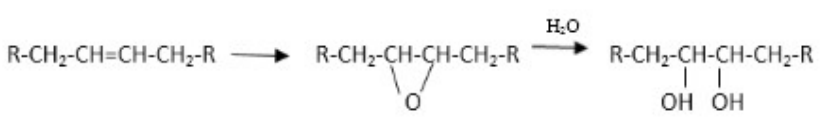

Figura 3. Esquema da reação de produção do diol

A reação se processou por 14 horas e, ao longo desse tempo, foram retiradas alíquotas após 3, 6, 9 e 14 horas de reação e obtidos os respectivos espectros de RMN H1 para se determinar o perfil da reação. Esse conjunto de espectros mostra que com apenas três horas de reação o diol já começou a ser formado e que em apenas nove horas 
de reação já seria suficiente para obter a quantidade de diol desejada. Que pode ser notado pelo perfil da reação apresentado abaixo, em que 9 horas se tem a maior produção do diol.

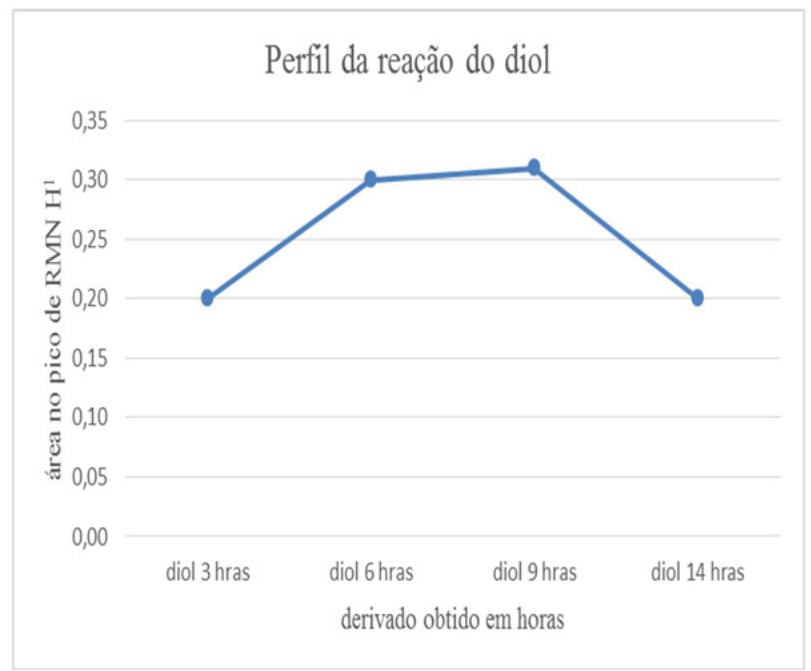

Figura 4. perfil da reação de produção do diol

O segundo derivado elaborado foi o diéster a partir do diol. Como descrito anteriormente, a reação durou 30 minutos. Foi feito o espectro de RMN H1 do produto obtido para atestar a formação do diéster.

\section{RESULTADOS DOS ENSAIOS FÍSICO- QUÍMICOS}

O primeiro ensaio realizado foi o Índice de Acidez Total (IAT). Os resultados estão na tabela 2.

Tabela 2. Resultados do Índice de Acidez Total

\begin{tabular}{|c|c|c|c|}
\hline Amostra & $\begin{array}{c}\text { Volume } \mathrm{NaOH} \\
(\mathrm{mL})\end{array}$ & $\begin{array}{c}\text { massa do óleo } \\
(\mathrm{g})\end{array}$ & IAT \\
\hline óleo de Canola & 0,2 & 2,078 & 0,055 \\
\hline diol & 1,5 & 2,023 & 0,414 \\
\hline diéster & 10 & 2,055 & 2,761 \\
\hline
\end{tabular}

Os resultados para o Índice de Acidez Total mostraram que a acidez do óleo aumentou para os derivados. Isto se deve ao fato de a reação de epoxidação necessitar de quantidade significativa de ácido fórmico, e mesmo com a separação e secagem do óleo, o mesmo ainda permanece com maior acidez que anteriormente. Porém a reação de produção do diéster ocorre em meio menos ácido que a epoxidação e produção do diol, e mesmo assim o produto final apresenta uma acidez muito elevada em comparação com o diol.

A viscosidade cinemática foi medida para o óleo de Canola e seus dois derivados. A tabela 3 apresenta os dados para as três espécies químicas.

Tabela 3. Resultados da viscosidade cinemática

\begin{tabular}{|c|c|c|c|}
\hline Amostra & $\begin{array}{c}\text { Visc 40 } \\
(\mathrm{mm} 2 / \mathrm{s})\end{array}$ & $\begin{array}{c}\text { Visc 100 } \\
(\mathrm{mm} 2 / \mathrm{s})\end{array}$ & IV \\
\hline óleo de Canola & 36,51 & 8,25 & 211 \\
\hline diol & 213,70 & 23,79 & 138 \\
\hline diéster & 209,00 & 23,44 & 138 \\
\hline
\end{tabular}

Observa-se um aumento de viscosidade de ambos os derivados com relação ao óleo de Canola in natura, devido ao menor número de insaturações presentes nos derivados. Por sua vez, o índice de viscosidade do óleo de Canola in natura foi maior que dos derivados, contudo todos são ainda superiores ao da maioria dos óleos lubrificantes derivados de petróleo.

\section{Conclusões}

Os resultados obtidos permitiram avaliar o potencial do óleo de Canola in natura e de seus dois derivados para atuarem como óleo lubrificante. Dos resultados apresentados, pode-se confirmar a produção dos derivados do óleo de canola, tanto o diol quanto o diéster, por meio do RMN H1, já que estes eram a base para o desenvolvimento do trabalho. A reação de epoxidação gerou bons resultados de maneira que a reação de produção do diol pôde ser acompanhada. 
Analisando-se o perfil da reação de produção do diol apresentado conclui-se que se teve uma boa produção do derivado entre seis e nove horas de reação e que a mesma sofreu um decaimento após as nove horas. Concluindo-se então que seriam necessárias menos que as quatorze horas totais para a obtenção da quantidade desejada do diol.

Outro resultado interessante se diz a respeito da viscosidade cinemática que aumentou muito do óleo in natura para os derivados, sendo mais elevada que a maioria dos óleos lubrificantes de bases minerais analisados. O índice de viscosidade mesmo sendo menor nos derivados, ainda assim é mais elevado que nos lubrificantes de petróleo.

Tudo isso nos leva a bons resultados de viscosidade, característica fundamental para um bom óleo lubrificante. Confirmando o que foi afirmado por Borugadda, Somidi e Dalai (2017)[10] que devido a abertura do anel dos epóxidos os óleos vegetais passam a oferecer funcionalidades interessantes como fluidez melhorada a baixa temperatura, viscosidade melhorada e alta estabilidade, atuando como bons lubrificantes.

\section{Referências Bibliográficas}

1. Antoniassi, Rosemar; Freitas, Sidinea Cordeiro. Árvore Do Conhecimento - Tecnologia de Alimentos. Disponível em: $<\mathrm{http}: / /$ www.agencia.cnptia.embrapa.br/gestor/tecnologia_de_alimentos/ arvore/CONT000gc8yujq302wx5ok01dx91cx1g7v3u.html>. Acesso em: 02 abr. 2017.

2. Definição e histórico de Canola. Disponível em: $<$ http://www.cnpt. embrapa.br/culturas/canola/definicao.htm>. Acesso em: 02 abr. 2017.

3. Kamalakar, Kotte, et al S.L.. Vegetable Oil-Based Ethanolamides as Potential AntiOxidant Additives for Lubricant Formulations. Industrial \& Engineering Chemistry Research. 17-Nov-2016. 26 p.

4. Matos, Paulo Roberto Rodrigues. Utilização de óleos vegetais como bases lubrificantes. 2011. 125 p. Dissertação de mestrado (Química)- Instituto de Química, Universidade de Brasília, [S.l.], 2011. Disponível em: <http://repositorio.unb.br/ bitstream/10482/8755/1/2011_PauloRobertoRodriguesdeMatos.pdf>. Acesso em: 02 ago. 2016.

5. Okieimen, F.E.; Pavithran, C.; Bakare, I.O. Epoxidation and hydroxlation of rubber seed oil: One-pot multi-step reactions. Eur J Lipid Sci Technol. 2005;107(5):330-6.

6. Origem e usos da canola. Março, 2014. Disponível em: <http://www. cnpt.embrapa.br/biblio/do/p_do149_2.htm>. Acesso em: 02 abr. 2017.

7. Souza, Líria Alves. Composição dos derivados do petróleo. Disponível em: <http://mundoeducacao.bol.uol.com.br/quimica/ composicao-dos-derivados-petroleo.htm>. Acesso em: 02 abr. 2017

8. Mcnutt, Josiah; Sophia He, Quan. Development of biolubricants from vegetable oils via chemical modification. Journal of Industrial and Engineering Chemistry, Canadá, 21 fev. 2016. p. 36. Disponível em: <http://www.elsevier.com /locate/jiec >. Acesso em: 19 jul. 2017.

9. Erhan, Sevim Z. et al. Lubricant Base Stock Potential of Chemically Modified Vegetable Oils. Journal of agricutural and food chemistry, Pensilvania, 19 jul. 2008. American Chemical Society, p. 56.

10. Boruggada, Venu Babu; Somidi, Asish K. R.; Dalai, Ajay K. Chemical/Structural Modification of Canola Oil and Canola Biodiesel: Kinetic Studies and Biodegradability of the Alkoxides . 2017. 14 p. Artigo (Catalysis and Chemical Reaction Engineering Laboratories,)- University of Saskatchewan, Canadá, 2017.

11. Aandrade, Carlos Kleber Zago de. Preparação de Lactonas Via Formação de Ligação Carbono-Carbono. Sínteses Formais de (-)-Serriconina, (+)-Lactona de Prelog-Djerassi e Estudos Visando a Síntese do (+)-10-Desoximetinolídeo . 1996. 14 p. tese de doutorado (Química Orgânica)- UniCamp, [S.1.], 1996.

12. Miyake, Y; Yokomizo, K; Matsuzaki, N. Determination of unsaturated fatty acid composition by high-resolution nuclear magnetic resonance spectroscopy. J Am Oil Chem Soc. 1998;75(12):1091-4.

13. Zenebon, Odair ; Pascuet, Neus Sadocco; Tigela, Paulo. Métodos físico-químicos para análise de alimentos. Instituto Adolfo Lutz, São Paulo, 2008. 4 ed,. 1020 p.
${ }^{1}$ Laboratório de Ensino de Físico-Química (LEFQ) - Instituto de Química - Universidade de Brasília - C.P 4478, Brasília, DF, Brasil.

${ }^{2}$ Laboratório de Química Computacional (LQC) - Instituto de Química Universidade de Brasília - C.P 4478, Brasília, DF, 70910900, Brasil.

${ }^{3}$ Centro de Pesquisas e Análises Tecnológicas (CPT) - Agência Nacional de Petróleo, Gás Natural e Biocombustíveis (ANP), Brasília, DF, 70830902, Brasil.

*E-mail: politi@unb.br 